\title{
L'Atala de Chateaubriand et l'Atala de Girodet : la beauté de la mort
}

\section{Chiara Savettieri}

\section{Q OpenEdition}

\section{Journals}

\section{Édition électronique}

URL : http://journals.openedition.org/rief/858

DOI : $10.4000 /$ rief.858

ISSN : 2240-7456

\section{Éditeur}

Seminario di filologia francese

\section{Référence électronique}

Chiara Savettieri, «L'Atala de Chateaubriand et l'Atala de Girodet : la beauté de la mort », Revue italienne d'études françaises [En ligne], 2 | 2012, mis en ligne le 15 décembre 2012, consulté le 19 avril 2019. URL : http://journals.openedition.org/rief/858 ; DOI : 10.4000/rief.858

Ce document a été généré automatiquement le 19 avril 2019

\section{(c) (i) (9)}

Les contenus de la RIEF sont mis à disposition selon les termes de la Licence Creative Commons Attribution - Pas d'Utilisation Commerciale - Pas de Modification 4.0 International. 


\section{L'Atala de Chateaubriand et l'Atala de Girodet : la beauté de la mort}

\section{Chiara Savettieri}

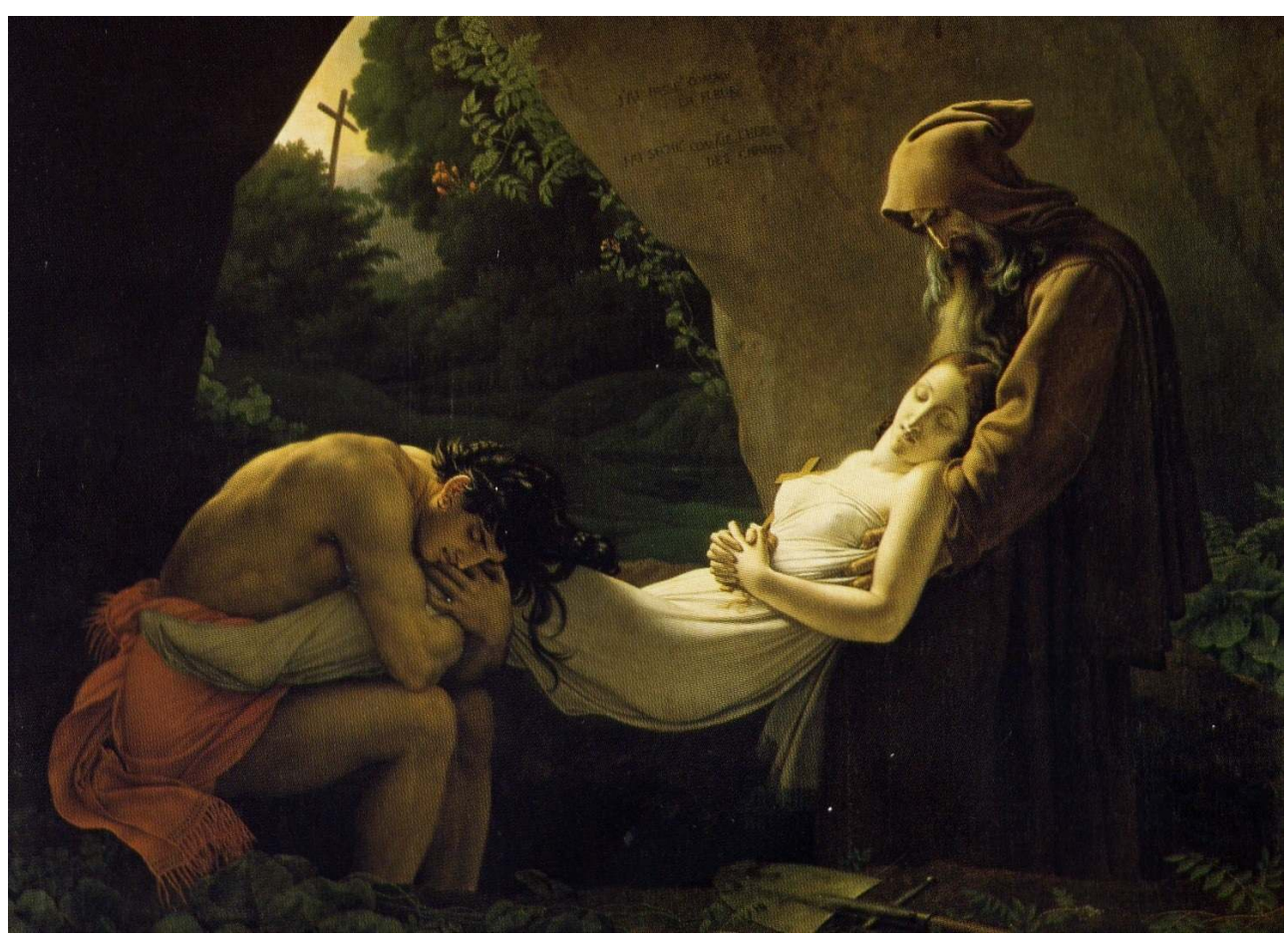

Anne-Louis Girodet-Trioson, Atala au tombeau, 1808. Paris, Musée du Louvre.

\section{Anne-Louis Girodet : le peintre de l'ut pictura poesis}

Vous avez déjà touché, Messieurs, le touchant épisode du Génie du christianisme, qui fournit à M. Girodet l'occasion, périlleuse pour tout autre, de se mesurer sur le même terrain avec le chantre d'Atala, et aussi le rare avantage de faire douter entre les deux ouvrages, lequel auroit le plus contribué à la célébrité de l'autre... Comment savoir en effet qui a le plus d'obligation à l'autre, du peintre qui doit au 
poète l'idée d'un sujet heureux, ou du poète dont le peintre sait revêtir les fictions de ce charme qui leur donne, avec les couleurs de la vie, le privilège de prendre rang, si l'on peut dire, parmi les êtres réels ?'

1 C'est ainsi qu'Antoine Chrysostome Quatremère de Quincy unit de façon indissoluble l' Atala de Chateaubriand au chef-d'œuvre d'Anne-Louis Girodet exposé au Salon de 1808, l' Atala au tombeau'.

Ce tableau, de même que la Maddalena Penitente d'Antonio Canova, exposée au même salon, correspondait à la nouvelle sensibilité religieuse que le Génie du Christianisme avait contribué à stimuler en Europe, désormais fatiguée du laïcisme révolutionnaire. Ce fut Louis François Bertin, dit Bertin l'Aîné, directeur avec son frère du Journal des débats, devenu par décret de Napoléon Journal de l'Empire en 1805, qui commanda à Girodet un tableau inspiré par le récit de Chateaubriand Atala ou les amours de deux sauvages dans le désert ${ }^{3}$. Ce texte, tiré d'un manuscrit conçu en Amérique, publié le 2 avril 1801, fut englobé par l'écrivain dans le Génie (1802), et réédité plusieurs fois. C'est très probablement chez Bertin ou chez son frère que le peintre avait rencontré l'écrivain vers 1804-1805. Girodet se confrontant avec ce sujet était bien conscient de répondre aux attentes du public, et en même temps d'exposer sa peinture à une inévitable et périlleuse comparaison avec un texte littéraire à grand succès, dont la prose avait une grande qualité picturale ${ }^{5}$. Or, cette comparaison était sans doute très stimulante pour Girodet : parmi les peintres de sa génération, il était celui qui cultivait et défendait le plus l'ut pictura poesis.

Deux aspects caractérisaient sa personnalité artistique : la quête de l'originalité, une obsession qui le poursuivit pendant toute sa carrière ; sa volonté de se présenter comme un peintre-poète, non seulement en tant qu'auteur lui-même d'un poème et de nombreux textes sur la peinture, et ami de plusieurs hommes de lettres (Jacques Delille, Bernardin de Saint-Pierre, Jean-François Ducis, Charles Hubert Millevoye, Alfred de Vigny), mais aussi parce qu'il chercha, à travers ses tableaux et ses écrits, à revendiquer pour la peinture des droits que les critiques de l'époque, influencés par le Laocoon (1767) de Lessing, traduit en français en 1802 , n'accordaient qu'à la littérature ${ }^{6}$ : la possibilité que la peinture exprime des idées, des concepts abstraits, qu'elle utilise des sujets merveilleux, qu'elle puisse représenter des situations dramatiques demandant des expressions très chargées. Girodet lutta contre la nette distinction entre les domaines littéraire et pictural, théorisée par Lessing, qui accordait au premier la liberté de traiter tous les sujets, même les plus terribles et expressifs, de se confronter avec la gamme la plus étendue de sentiments, et limitait en revanche le deuxième à la représentation de la beauté.

Deux ouvrages précédant l'Atala sont de véritables démonstrations de ces aspects. Dans l' Ossian (1801), l'artiste se lança dans la représentation des héros ossianiques qui rencontraient les ombres des généraux français morts pour la France. Ce mélange inédit de portraits de personnages réels avec des figures fictives, de même que la totale invraisemblance de la scène suscitèrent beaucoup de critiques. Dans une lettre à Bernardin de Saint-Pierre (post 1802-ante 1806), Girodet ne fait que revendiquer son originalité pour avoir représenté ce paradis celtique d'Ossian fait de brouillard sans suivre aucun modèle, ni tiré de la nature ni tiré d'autres œuvres artistiques, mais il ajoute que le peintre comme le poète a le droit de traiter des sujets merveilleux qui n'existent pas dans la réalité7. Dans le Déluge (1806) il osa peindre une famille dont la survie dépend entièrement d'une branche qui est sur le point de se rompre: un sujet très audacieux, même scandaleux, pour ceux qui partageaient les principes du Laocoon. En effet Lessing, d'une part, interdisait aux artistes figuratifs les sujets trop transitoires, car ils ne seraient 
pas conformes à l'identité « spatiale » et non temporelle de la peinture et de la sculpture ; d'autre part, il condamnait les sujets trop terribles et violents dans la peinture car ils entrainaient une expressivité trop poussée, non adaptée aux arts visuels qui devaient se restreindre au beau, mais tout à fait légitime en poésie, art du temps, car tout instant trop intense et chargé d'émotivité y serait dépassé par un moment plus serein ${ }^{8}$. C'était la fixité des arts visuels qui les rendait aux yeux de Lessing incompatibles avec la représentation des passions et des sentiments les plus forts, et en bornaient le champ d'action au domaine de la beauté. Contre l'auteur allemand Girodet choisit de peindre un instant très transitoire (la branche qui se casse) et exhiba sur le visage du père la représentation de l'effroi : « Adieu beauté idéale », tel fut l'éloquent commentaire de David à cet ouvrage9.

5 D'après cette prémisse il est évident que pour un peintre comme Girodet, si convaincu de l'ut pictura poesis, se mesurer avec le texte de Chateaubriand représentait une opportunité de plus pour affirmer encore à nouveau la possibilité d'ouvrir les confins entre peinture et littérature que Lessing avait fermés à double tour.

\section{L'Atala de Chateaubriand ou le Triomphe de la Mort}

6 Atala, fille d'un Espagnol et d'une Indienne, vit dans la tribu de sa mère dans une forêt de l'Amérique du Nord. Chactas, jeune Natchez, est prisonnier et condamné à mort, mais Atala le délivre et, amoureux, ils s'échappent. Cependant la jeune fille ne peut pas s'unir avec Chactas à cause d'un vœu de chasteté que sa mère lui avait fait prononcer avant de mourir, mais qu'elle avait en réalité conçu déjà à la naissance d'Atala lorsque la petite était en danger de mort. Craignant de tomber dans la tentation, Atala décide de se suicider : pendant une tempête elle avale un poison mortel à l'insu de son amant. Ensuite le couple rencontre un missionnaire, le père Aubry qui, apprenant leur histoire, décide de les marier; mais bientôt les effets du poison se manifestent et Atala confesse le vœu et son acte. Chactas est atterré tandis qu'Aubry explique à la fille que le vœu pouvait être annulé par une autorité de l'Église et que le suicide est un péché mortel, tout en la consolant par la miséricorde de Dieu, qui lui pardonnera sa naïveté et son ignorance. Atala meurt soulagée par ces mots. L'histoire est racontée rétrospectivement par Chactas âgé à René, un Français accueilli par la tribu des Nachtez.

7 Chateaubriand, qui inséra le récit dans le Génie pour montrer les "harmonies de la Religion chrétienne avec les scènes de la nature et les passions du cœur humain $»^{10}$, présente Atala comme l'emblème du péché inconscient et de la rédemption, de la passion amoureuse et de la soumission aveugle à la religion; elle est l'image de la mort comme réconciliation avec le Divin, la personnification d'une pureté sacrale qui reste pour son amant l'objet d'un désir destiné à rester insatisfait. Atala est sensuelle et candide, désirable, mais chaste dans la mort qui l'enlève au monde.

La narration, toute imprégnée par le sentiment de mélancolie et de regret éprouvé par Chactas rappelant l'histoire, est rythmée de façon obsessionnelle par des images et des expressions liées à la mort qui, laissant entrevoir la conclusion tragique, s'intensifient au fur et à mesure qu'on s'approche de la fin où thanatos triomphe. Dans l'épilogue, en effet, le lecteur découvre que plusieurs années après les faits reportés par Chactas à René, ces derniers (l'indien adoptera l'européen) seront massacrés par les Français et qu'au père Aubry aussi sera réservée une fin violente de martyre. Le sommet de ce climax funèbre est l'image touchante du nouveau-né mort de la petite fille de René, qui a accouché dans la douleur pendant la marche d'exil de sa tribu ${ }^{11}$. Celle-ci amène aussi dans ce voyage les 
cendres de Chactas, de René, d'Atala et du père Aubry (l'indien avait retrouvé les restes de ces dernières et les avait conservées avec lui): après de nombreuses péripéties, les protagonistes sont ainsi finalement réunis.

9 Tout au long de la narration, la mort est présentée sous son double visage : une affreuse souffrance pour celui qui reste, une libération des impuretés de la vie, un apaisement, une rédemption pour celle qui meurt. Parcourons donc quelques-unes de ces images de la mort qui rythment le texte.

$10 \mathrm{Au}$ début Chactas, après avoir révélé à René que, encore très jeune, il vit son père mourir dans une guerre entre tribus, tandis que lui fut blessé deux fois, déclare : «Oh! Que ne descendis-je alors dans les pays des âmes! J'aurais évité les malheurs qui m'attendaient sur la terre $»^{12}$. Cette déclaration jette une lumière sombre sur le reste du récit, révélant le point de vue du narrateur qui invoque la mort pour faire cesser ses souffrances.

11 Ensuite Chactas raconte sa première rencontre avec Atala comme une sorte d'apparition céleste :

Elle joignait à cela des grâces plus tendres : une extrême sensibilité, unie à une mélancolie profonde, respirait à ses regards; son sourire était céleste. Je crus que c'était la Vierge des derniers amours, cette vierge qu'on envoie au prisonnier de guerre pour enchanter sa tombe. (ARL, p. 105)

12 Atala a un charme sacral, lié dès le début à l'idée de la mort qui représente le véritable leitmotiv du récit: Chactas croit voir en elle la vierge qui était envoyée aux prisonniers pour "enchanter" leur tombeau. Atala mourant le rappellera à son amant en l'interprétant comme un présage de sa mort ${ }^{13}$. Après le premier baiser, un pressentiment funèbre s'insinue dans le récit : «Beau prisonnier, - lui dit Atala - j'ai follement cédé à ton désir; mais où nous conduira cette passion? Ma religion me sépare de toi pour toujours » (ARL, p. 108).

13 Pendant la fuite, les amants rencontrent une mère qui, veillant le tombeau de son nouveau-né, leur dit que cette mort prématurée a préservé son enfant des souffrances de la vie: «Heureux ceux qui meurent au berceau! ils n'ont connu que les baisers et les souris d'une mère! » (ARL, p. 112).

14 Lorsque Chactas contemple Atala qui prie, son image lui apparait si sacrale qu'il lui semble que la jeune fille va bientôt s'envoler vers le ciel, ce qui lui fait pressentir que la disparition de son amante est proche :

Ah! qu'elle me parut divine, la simple Sauvage, l'ignorante Atala, qui à genoux devant un vieux pin tombé, comme au pied d'un autel, offrait à son Dieu, à travers la cime des bois, ses vœux pour un amant idolâtre! Ses yeux levés vers l'astre de la nuit, ses joues brillantes des pleurs de la religion et de l'amour, étaient d'une beauté immortelle. Plusieurs fois il me sembla qu'elle allait prendre son vol vers les cieux; plusieurs fois je crus voir descendre sur les rayons de la lune et entendre dans les branches des arbres, ces Génies que le Dieu des chrétiens envoie aux ermites des rochers, lorsqu'il se dispose à les rappeler à lui. J'en fus affligé, car je craignis qu'Atala n'eût que peu de temps à passer sur la terre. (ARL, p. 112-113) ${ }^{14}$

15 Lorsqu'Atala libère Chactas, emprisonné une deuxième fois, elle apparaît au gardien du prisonnier comme un fantôme, l'Esprit des ruines. Pendant la deuxième fuite, Atala lui déclare son amour et en même temps lui enlève tout espoir, se révélant ainsi comme un être mystérieux et insaisissable ${ }^{15}$ :

O mon jeune amant! Je t'aime comme l'ombre des bois au milieu du jour! Tu es beau comme le désert avec toutes ses fleurs et toutes ses brises [...] si ma main 
tombe sur la tienne, il me semble que je vais mourir [...]. Eh ! bien, pauvre Chactas, je ne serai jamais ton épouse! (ARL, p. 122) a deur rencontre : Chactas, effrayé, soupçonne la mort de son amante, implorant le missionnaire «Ô vous, que le ciel accompagne et fortifie, pénétrez dans ces ombres » ( ARL, p. 139). mes lèvres tremblantes, tu ne savais pas! Tu ne savais pas que tu n'embrassais que la mort! » (ARL, p. 145). La mort semble ensuite presque les réunir tous les trois, anticipant ainsi la réunion de leurs cendres de l'épilogue: «Le flambeau échappe de la main du Solitaire, je tombe mourant près de la fille de Lopez, le vieillard nous saisit l'un et l'autre dans ses bras, et tous trois, dans l'ombre, nous mêlons un moment nos sanglots sur cette couche funèbre $»^{17}$.

Aubry convainc Atala que la vie n'est qu'une souffrance universelle - «l'habitant de la cabane, et celui des palais, tout souffre, tout gémit ici-bas» (ARL, p. 147-148) - et lui représente l'image de la Vierge Marie qui l'invite aux cieux pour se reposer auprès de Jésus-Christ et profiter pour toujours des joies célestes ${ }^{18}$ : Atala est assimilée à une créature céleste.

20 Aubry et Chactas décident de transporter ses restes dans une grotte où ils la veilleront toute la nuit pour l'ensevelir ensuite sous une arche de rochers. Pendant la veille Atala est ainsi décrite :

Atala était couchée sur un gazon de sensitives de montagnes; ses pieds, sa tête, ses épaules et une partie de son sein étaient découverts. On voyait dans ses cheveux une fleur de magnolia fanée ... celle-là même que j’avais déposée sur le lit de la vierge pour la rendre féconde. Ses lèvres, comme un bouton de rose cueilli depuis deux matins, semblaient languir et sourire. Dans ses joues, d'une blancheur éclatante, on distinguait quelques veines bleues. Ses beaux yeux étaient fermés, ses pieds modestes étaient joints, et ses mains d'albâtre pressaient sur son cœur un crucifix d'ébène; le scapulaire de ses vœux étaient passé à son cou. Elle paraissait enchantée par l'Ange de la mélancolie, et par le double sommeil de l'innocence et de la tombe . Je n'ai rien vu de plus céleste. Quiconque eut ignoré que cette jeune vestale avait joui de la lumière, aurait pu la prendre pour la statue de la Virginité endormie. Le religieux ne cessa de prier toute la nuit. J'étais assis en silence au chevet du lit funèbre de mon Atala. Que de fois, durant son sommeil, j'avais supporté sur mes genoux cette tête charmante! Que de fois je m'étais penché sur elle, pour entendre et pour respirer son souffle! Mais à présent aucun bruit ne sortait de ce sein immobile, et c'était en vain que j'attendais le réveil de la beauté ! La lune prêta son pâle flambeau à cette veillée funeste. Elle se leva au milieu de la nuit, comme une blanche vestale qui vient pleurer sur le cercueil d'une compagne. Bientôt elle répandit dans les bois ce grand secret de mélancolie, qu'elle aime à raconter aux vieux chênes, et aux rivages antiques des mers. De temps en temps, le religieux plongeait un rameau fleuri dans une eau consacrée; puis secouant la branche humide, il parfumait la nuit des baumes du ciel. Parfois il répétait sur un air antique quelques vers d'un vieux poète nommé Job ; il disait «j'ai passé comme une fleur ; j'ai séché comme l'herbe des champs. Pourquoi la lumière a-t-elle été donnée à un misérable, et la vie à ceux qui sont dans l'amertume du cœur? » (ARL, p. 155-156) ${ }^{19}$

Atala est enveloppée d'une aura d'innocence et de béatitude, et en même temps de sensualité. Pourquoi Chateaubriand la compare-t-il à une statue de la Virginité endormie ? 
Pourquoi cette référence à l'art statuaire? Dans le Génie du Christianisme, il souligne l'incompatibilité entre la sculpture et la représentation des cadavres ${ }^{20}$, envisageant plutôt que, dans les sépulcres, soient représentés «d'un côté, les pleurs de la famille et les regrets des hommes; de l'autre le sourire de l'espérance et les joies célestes [...] La mort pourrait y paraître, mais sous les traits d'un ange à la fois doux et sévère... $»^{21}$. En outre, dans une note de l'édition de 1803, il rappelle que le marbre "offrant des formes palpables et glacées, ressemble trop à la vérité ${ }^{22}$ et n'est donc pas adapté à peindre la mort. C'est pourquoi l'écrivain conçoit l'image d'une belle morte sous la forme d'une sculpture, un art dans lequel la mort ne peut être représentée que de façon métaphorique: comme un sommeil. Une vision classiciste de la statuaire émerge ici comme quoi l'art ne doit pas représenter des images hideuses ou terribles ${ }^{23}$. Vision qui est en parfaite conformité avec les idées de Winckelmann et de Lessing, lequel, dans la dissertation publiée à Berlin en 1769, Wie die Alten den Tod gebildet ?, affirmait que les Grecs avaient eu une âme trop élevée pour concevoir la représentation de la mort de façon terrible ${ }^{24}$, un argument déjà avancé dans une note du Laocoon ${ }^{25}$. Mais Chateaubriand s'inspire aussi des réflexions sur les tombeaux de Bernardin de Saint-Pierre ${ }^{26}$ qui, dans les Études sur la nature, souligne que ceux-ci devraient offrir une image sereine et consolatrice de la mort et repousser tout ce qui pourrait en rappeler l'atrocité à travers l'utilisation d'allégories :

Il n'y auroit sur ces tombeaux ni squelettes, ni ailes de chauves-souris, ni faux du Temps, ni aucun de ces attributs effrayans avec lesquels nos éducations d'esclaves cherchent à nous faire peur de la mort, de dernier bienfait de la nature ; mais on y verroit les symboles qui annoncent une vie heureuse et immortelle; des vaisseaux battus de la tempête qui arrivent au port, des colombes qui prennent leurs vol vers les cieux...27

Avec l'expression statue de la Virginité endormie, et donc à travers la référence à l'art statuaire, Chateaubriand peut unir l'impossible : la mort et la beauté, ou mieux rendre la mort belle et comme un objet de désir, sublimer thanatos dans l'art du marbre. D'ailleurs, Chactas nomme plusieurs fois Atala morte «la beauté » comme si elle en était la personnification.

\section{L'Atala de Girodet entre eros et thanatos, entre la vie et la mort}

Girodet a choisi de représenter l'enterrement d'Atala, un moment de l'histoire sur lequel Chateaubriand ne donne pas beaucoup de précisions. Le père Aubry et Chactas sont sur le point d'ensevelir Atala sous l'arche d'un rocher. La jeune fille, enveloppée du lin blanc d'Aubry, tient une croix dans ses mains; son visage est serein, la beauté de ses traits réguliers et de son corps harmonieux est révélée par la douce lumière de l'aube, qui rappelle l'Endymion peint par Girodet à Rome en 1792. Si, dans ce tableau, le peintre avait allié hypnos, eros et beauté idéale en s'inspirant de la douce pureté des marbres d'Antonio Canova $^{28}$, dans Atala il relie tous ces éléments à thanathos. Or, cette beauté d'Atala correspond parfaitement aux canons de la beauté idéale théorisée par Winckelmann ${ }^{29}$ : une beauté dans laquelle le calme, la simplicité, l'absence de caractères trop marqués triomphent, et de laquelle un charme sensuel se dégage. C'est ce qui se passe dans l'Atala de Girodet comme le soulignèrent de nombreux critiques de l'époque, remarquant que la protagoniste est si belle qu'elle semble dormir : «M. Buteux trouve, comme bien d'autres, 
qu'Atala ne paraît pas assez morte $»^{30}$, «Atala paroit plutôt endormie que morte $»^{31}$. Le journaliste du Courrier de l'Europe insinue que le charme de la jeune fille suscite chez le spectateur le désir morbide de la réveiller : Atala «n'est point morte, bien loin de donner la pensée de prier pour elle, elle provoque la maligne envie de la réveiller et de rendre jaloux le pauvre Chactas, qui réellement a l'air bien affligé $»^{32}$. Pour le salonnier du Publiciste, "Atala paraît plutôt endormie que morte, ce qui rend équivoque l'action des deux autres personnages, et pourrait faire naître des idées révoltantes $»^{33}$. Selon David, si "la tête, le coloris d'Atala appartiennent bien à une morte ", « les mains ne sont-elles pas mortes? $\aleph^{34}$. D'autres considérèrent de façon positive cette ambiguïté sommeil-mort: d'après Julie Candeille, Girodet avait donné « la vie au tombeau /Et des grâces à la mort même $»^{35}$.

Cette figure d'Atala, dans sa candeur presque aveuglante, unit beauté et sensualité comme les statues de Canova, artiste que Girodet appréciait beaucoup ${ }^{36}$ : en effet elle ressemble à une créature de marbre, belle et désirable comme les œuvres du sculpteur italien, qui parvenaient à susciter chez le spectateur le désir de les embrasser ${ }^{37}$. Avec cette référence à la sculpture, Girodet traduit la phrase de Chateaubriand qui peint Atala comme la statue de la Virginité endormie. Répondant aux remarques négatives suscitées par la beauté sensuelle d'Atala, après avoir souligné la fidélité de Girodet à la description que Chateaubriand fait d'Atala morte, Jean-Baptiste Boutard, chroniqueur des Salons au Journal de l'Empire, frère de l'épouse de Bertin l'Aîné et ami de Girodet ${ }^{38}$, rappelle de façon très pertinente :

Le peintre, en reculant de nos yeux ce que la mort a de signes plus effrayans, s'est permis un mensonge convenu dans les arts de l'imagination, et trop conforme au cœur de l'homme, pour qu'il ne soit point d'un usage universel. Les orateurs, et surtout les poètes, l'autorisent par une multitude d'exemples. ${ }^{39}$

Girodet a donné une image consolante et belle de cette jeune morte, suivant la même conception de la représentation de la mort dans les arts que celle exprimée par Chateaubriand dans le Génie, et condensée par la description d'Atala comme « la statue de la Vierge endormie ». Le peintre a donc en réalité opéré une crase entre la scène de la veille et celle de l'enterrement, ce qui est prouvé aussi par la phrase, répétée par Aubry pendant la veille et tirée du Livre de Job, «j'ai passé comme une fleur; j'ai séché comme l'herbe des champs ", que Girodet a représentée gravée sur le rocher. Le missionnaire, raconte Chateaubriand, gravait sur les troncs des arbres des vers tirés d'Homère et de la Bible: le peintre a transposé cet usage sur sa toile pour visualiser une phrase qui est fondamentale dans le récit, mais qui est prononcée et non pas gravée par le religieux. Il met ainsi en peinture ce qui est verbal et qui ne pourrait pas être communiqué au spectateur autrement. En effet la phrase gravée non seulement renvoie au moment de la veille, mais elle constitue aussi un commentaire à toute l'histoire: la précarité et la dureté de la vie et la mort comme fin des malheurs.

Compte tenu de toutes ces références à la veillée mortuaire, il est naturel de se demander pourquoi Girodet n'a pas choisi de représenter celle-ci plutôt que de fondre deux phases distinctes du récit. En fait cette fusion, pas tout à fait fidèle à la lettre du récit de Chateaubriand, lui permettait de saisir et de condenser avec le langage pictural les thèmes fondamentaux du texte. L'Atala de Chateaubriand est une créature sensuelle et sacrale en même temps : or la représentation de l'enterrement permettait à Girodet de souligner justement la sacralité de la protagoniste à travers la référence à des iconographies chrétiennes comme la mise au tombeau du Christ ou la lamentatio Christi, 
dont le Saint Sépulcre du sculpteur Ligier Richier à Saint-Michel ${ }^{40}$ constitue un superbe exemple. La position du corps de la vierge rappelle celle du Christ déposé et celle de Chactas ressemble à l'iconographie de la Madeleine accablée par la douleur de la mort de Jésus. La scène a aussi des ressemblances avec une autre iconographie religieuse: la Dormitio Virginis ${ }^{41}$. Le peintre a appliqué ces schémas iconographiques chrétiens à un sujet profane avec pour résultat de sacraliser Atala, tout en l'enveloppant d'un charme sensuel. C'est pourquoi à côté des critiques remarquant qu'Atala était trop belle et désirable pour être morte, d'autres observaient l'aura sacrale de la scène : « un silence religieux, produit par l'harmonie de tout ensemble, semble régner sur cette composition $»^{42}$.

Mais surtout le choix de mélanger veillée mortuaire et mise au tombeau offrait à Girodet la possibilité d'insister sur le leitmotiv du récit et de la plupart de la production littéraire de Chateaubriand: la pulsion de mort ${ }^{43}$. L'enterrement implique nécessairement la visualisation du tombeau et rappelle au spectateur toute la réalité et l'inéluctabilité de thanatos qui dans le tableau contraste avec la beauté de la protagoniste. Girodet non seulement met en valeur la représentation du tombeau, placé en bas et presque offert aux yeux du spectateur, mais y place tous les personnages : Aubry et Chactas sont dans le même tombeau qui va accueillir Atala. Ce choix iconographique incarne emblématiquement le fond du récit de Chateaubriand, le triomphe de la mort : cette mort, invoquée plusieurs fois par Chactas comme la seule solution aux maux de la vie, cette mort d'Atala annoncée pendant toute la narration, cette mort triomphant dans l'épilogue où les cendres de tous les protagonistes sont réunies, et qui est anticipée par le moment où s'étreignent les trois personnages après avoir découvert l'acte fatal d'Atala.

Mais il y a une autre crase: l'image de l'indien qui serre les genoux de son amante n'appartient ni à la section du récit concernant la veille mortuaire ni à celle de l'enterrement. C'est par contre d'une part un renvoi très clair à la fin du récit lorsque Chactas, avant de repartir, se rend au tombeau, et « enseveli dans la plus amère rêverie ", réfléchit sur la vanité de la vie ${ }^{44}$ : ensuite il tombe à genoux et étreint le tombeau en criant « Dors en paix dans cette terre étrangère, fille trop malheureuse ! Pour prix de ton amour, de ton exil et de ta mort, tu vas être abandonnée, même de Chactas!» (ARL, p. 159). D'autre part l'union de l'étreinte de deux amants et de l'image du tombeau rappelle le désir exprimé par Chactas, «brûlant de désir », pendant la fuite, de proposer à la vierge de «bâtir une hutte sur ces rivages et de nous y ensevelir ensemble » (ARL, p. 125) : Girodet visualise ainsi la superposition complexe d'eros et thanathos qui marque le récit. 


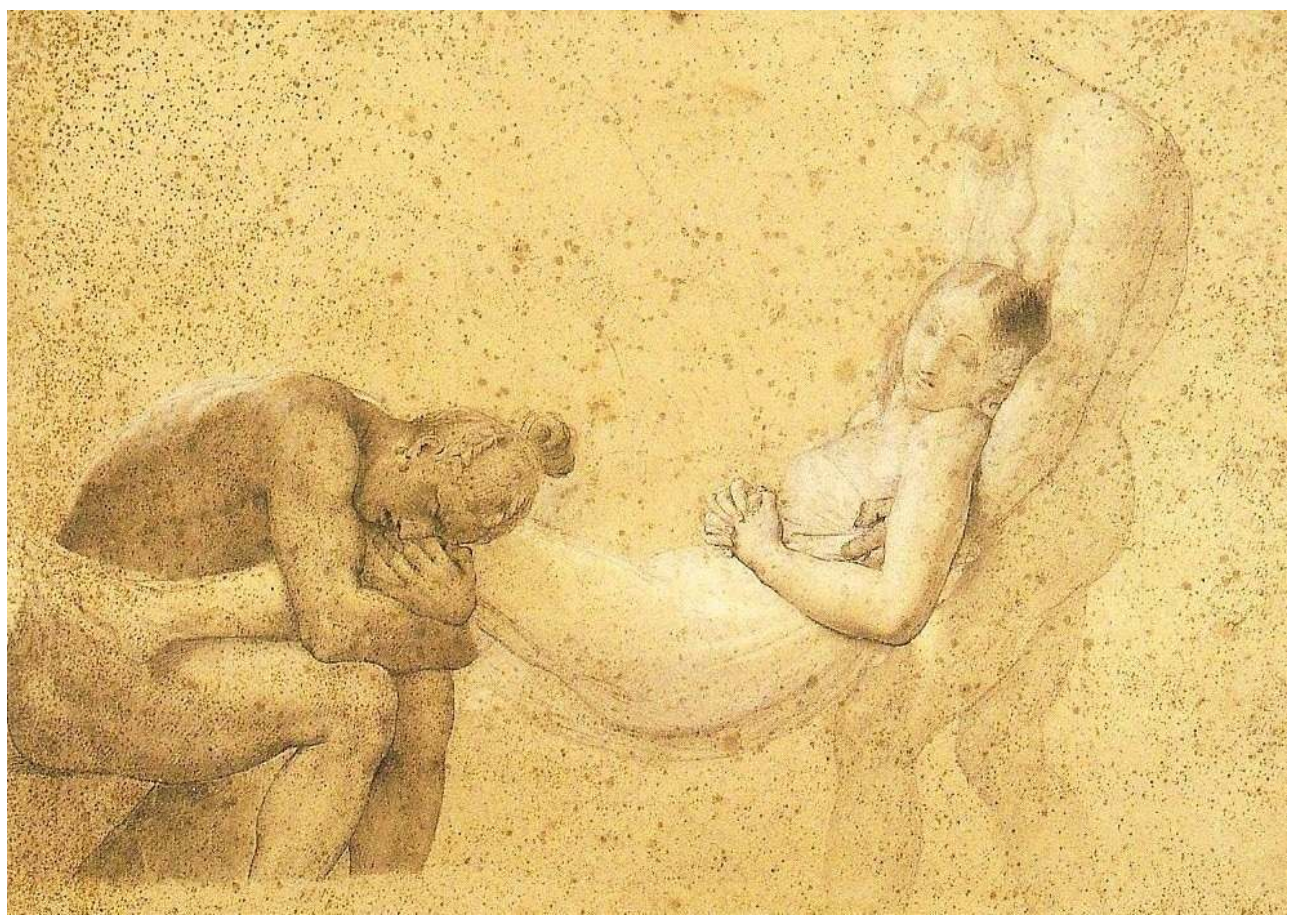

Anne-Louis Girodet-Trioson, Chactas embrassant les jambes d'Atala, pierre noire, estompe et rehauts de blanc sur papier beige, 42,8x59,5 cm. Paris, Musée du Louvre, Département des arts graphiques.

Le peintre, lecteur et ami de Bernardin de Saint-Pierre, de Jacques Delille et d'autres auteurs qui s'étaient confrontés aux thèmes de la mort et des tombeaux ${ }^{45}$, retrouvait dans la statue de la Virginité endormie de Chateaubriand l'image emblématique d'un mélange d' eros, hypnos et thanathos qui caractérise une bonne partie de sa production picturale. En effet souvent, dans la peinture de Girodet, la beauté n'atteint à l'idéal et à la pureté parfaite que dans l'inaction, l'évanouissement (la femme du Déluge), le sommeil (Endymion de 1791), en général dans des situations qui ressemblent à la mort, ou qui coïncident avec la mort comme c'est le cas de l'Atala, ou de la Galathée de 1819 (qui est entre la froide immobilité du marbre et le réveil), parce que la vie étant douleur, souffrance, donc expression, même outrée, s'avère incompatible avec la beauté (l'homme du Déluge, les grimaces des guerriers de la Révolte du Caire de 1810); en même temps, la mort devenant sous ses pinceaux beauté perd son caractère hideux et finit par ressembler au sommeil ( Atala, le pacha de la Révolte du Caire de 1810).

Mais Girodet fut si intrigué par la description d'Atala comme statue de la Virginité endormie, qu'il l'utilisa aussi dans ses écrits esthétiques, rédigés à partir de 1800-1801 ${ }^{46}$, comme exemple de grâce dans les arts visuels. Dans ses textes, Girodet présente la grâce comme un je ne sais quoi qui donne à la beauté un aspect attachant, charmant et même sensuel, sans lui enlever le côté idéal et pur : c'est un voile d'illusion de vie, une légère patine de nature enveloppant une beauté qui, étant idéale, n'appartient pas à ce monde, mais à un ailleurs métaphysique, à un non-être, et s'assimile donc à la mort ${ }^{47}$ (ce qui correspond au niveau pictural à cette coïncidence beauté idéale-mort). C'est pourquoi l'Atala de Chateaubriand, créature qui réunit beauté et mort, illusion de vie (sommeil) et pureté intacte qui échappe à la vie, devient pour lui l'icône de la grâce.

Si dans la deuxième veillée ${ }^{48}$ Girodet loue les artistes grecs qui faisaient allusion à la mort à travers des images allégoriques comme le sommeil, ce qui rejoint la position de 
Chateaubriand $^{49}$, dans la Sixième veillée il relie la grâce à la mort en reprenant la statue de la Virginité endormie de Chateaubriand :

Même à la grâce encore prêtant de saints attraits, La mort n'efface point, elle pâlit ses traits.

De cette vierge éteinte, au printemps de son âge, L'œil se dérobe au jour ; tel, d'un léger nuage Se couvre en palissant l'astre brillant des nuits ; Ses charmes sont voilés et ne sont pas détruits ; L'hyacinthe fleurit où fleurissaient les roses ; Remplaçant le corail sur ses lèvres mi-closes, La violette y verse une tendre pâleur, objet de doux regrets et non pas de terreur. Un calme solennel, des sens domptant la flamme, Sans oppresser son cœur semble assoupir son âme ; L'ange de l'espérance seul a fermé ses yeux : Attendant un réveil paisible et glorieux, Dans le sein maternel d'une déesse amie C'est la Virginité qui repose endormie. ${ }^{50}$

Cette image est proposée de nouveau dans la Dissertation sur l'amour et les grâces (1816 ca.) :

La grâce, don céleste, survit quelques instans à la vie elle-même dans l'être aimable qui en était orné. Voyez cette jeune beauté que vous eussiez prise de loin pour la statue de la pudeur qui sommeille: ses longues paupières ombragent, pour toujours, le tendre azur de ses yeux, aussi pur que l'azur du ciel ; à peine laissentelles entrevoir encore leur émail à demi effacé, semblable au faible et pâle croissant de la lune, prêt à disparaître dans les sombres nuages de la nuit. Son sein ne soulève plus, en le repoussant mollement, le chaste vêtement qui le retenait captif ; aucun souffle ne s'exhale de ces lèvres où venaient se confondre les soupirs discrets, les naïfs sourires du cœur; où affluaient les paroles expansives de la gaieté sans contrainte et de la félicité sans trouble ; mais elle est belle encore et d'une beauté toute sublime, dans ce calme solennel de tous sens. Non! Tant de grâces, tant de charmes et tant d'espérance ne peuvent être moissonnés pour toujours. La vierge de Naim [ ?] ${ }^{51}$ n'est point morte ; c'est la virginité qui repose, endormie dans les voiles mystérieux de l'éternité. $O$ vous, dont elle était l'orgueil et la joie ! Tendres et infortunés parens; amant jeune et vertueux dont elle devait bientôt, heureuse épouse, embellir la destinée; aimables compagnes avec qui elle échangeait ses innocentes confidences, et qui partagiez ses aimables jeux; contemplez encore un dernier moment cette jeune rose où elle venait s'épanouir, et dont le parfum, emblème de son âme céleste, doit lui survivre encore ! Donnez, donnez des pleurs à de si douloureux souvenirs! Mais qu'une pensée courageuse et consolante s'élève du sein même de vos regrets : la puissance inexorable qui vous l'a ravie, ne peut rien dans le séjour des âmes qu'elle habite et où vous vous rejoindrez un jour à elle, pour n'en être plus séparés. ${ }^{52}$

Girodet reconnut ainsi dans l'Atala de Chateaubriand son idéal de beauté - qui unit grâce et sublime, chasteté et sensualité, charme et mort. Mais avant tout, son interprétation picturale du récit de Chateabriand, fidèle plus à l'esprit qu'à la lettre, démontrait que la peinture pouvait non seulement se mesurer de façon originale avec un texte littéraire, mais aussi en saisir emblématiquement et efficacement les thèmes profonds: donc, encore une fois, ut pictura poesis.

Quatremère avait bien raison lorsqu'il soulignait qu'autant le texte de Chateaubriand avait contribué à la gloire de la toile de Girodet, autant ce tableau avait concouru à la renommée de l'ouvrage littéraire. Et d'ailleurs un grand salonnier comme Baudelaire avouait que l'Atala de Girodet était « quoiqu'en pensent certains farceurs qui seront tout à 
l'heure bien vieux, un drame de beaucoup supérieur à une foule de fadaises modernes innombrables $»^{53}$.

\section{NOTES}

1. A. C. Quatremère de Quincy, Éloge historique de M. Girodet lu à la séance publique de l'Académie royale des Beaux-arts le samedi $1^{\text {er }}$ octobre 1825, dans Recueil de Notices Historiques lues dans les séances publiques de l'Académie Royale des Beaux-arts à l'Institut par Quatremère de Quincy, Paris, A. Le Clere, 1834, p. 322-323.

2. H. Lemonnier, "L'Atala de Chateaubriand et l'Atala de Girodet", dans Gazette des Beaux-Arts, vol.LVI, mai 1914, p.363-371; G. Levitine, Girodet-Trioson: An Iconographical Study, Ph. D. Dissertation, Harvard University, 1953, Ann Arbor, New York, 1974, p. 263-7; Id., "Some unexplored aspects of the illustrations of Atala: the surenchères visuelles of Girodet and Hersent ", dans R. Switzer (dir.), Chateaubriand, Actes du Congrès de Wisconsin, 1968, Genève, Droz, 1970, p. 139-144; D. Wakefield, «Chateaubriand's Atala as a source of inspiration in nineteenth century art ", dans The Burlington Magazine, janvier 1978, p. 13-22 ; S. Nevison Brown, Girodet: a contradictory career, Ph. D. Dissertation, London University, 1980, p. 234 et sq; S. Bellanger, "Girodet et la littérature, Chateaubriand et la peinture », dans M. Fumaroli (dir.), Chateaubriand et les Arts, Paris, éditions de Fallois, 1999, p.111-136; Id., "Ut pictura poesis : Chateaubriand et les Arts ", Ibid., p. 11-42 ; S. Guégan, « De Chateaubriand à Girodet : Atala ou la belle morte", Ibid., p. 137-152 ; J. F. Moffitt, "The native american "sauvage" as pictured by french romantic artists and writers", dans Gazette des Beaux-arts, n. 141, septembre 1999, p. 123-126; S. Bellanger, "Le pathétique chrétien. Atala au tombeau», notice n. 51, dans S. Bellanger (dir.), Girodet. 1767-1824, Catalogue Exposition Louvre, Paris, Gallimard, 2005, p. 300-307.

3. D. Ternois, Ingres. Le portrait de Monsieur Bertin, Paris, Réunion des musées nationaux, "Solo », 1998, p. 10.

4. Bertin joua le rôle d'intermédiaire entre Chateaubriand et Girodet lorsque l'écrivain commanda son portrait, que le peintre réalisa pendant l'été de 1808 et qu'il exposa au Salon de 1810 (Portrait de Chateaubriand dit aussi Un homme méditant sur les ruines de Rome, Saint-Malo, Musée d'Histoire et d'Ethnographie).

5. A. France, «Image et structure dans Atala », dans Revue Romane, X, novembre 1975, p. 367-372.

6. Cf. C. Savettieri, Ingannare la morte. Anne-Louis Girodet-Trioson e l'illusione dell'arte, Palermo, Aesthetica, 2005, p. 10-12.

7. A.-L. Girodet, Correspondance, dans P. A. Coupin, Euvres posthumes de Girodet, Paris, Renouard, 1829, II, p. 277.

8. G. E. Lessing, Laokoon, 1766, trad. française Du Laocoon ou des limites de la poésie et de la peinture, Paris, Renouard, 1802, p. 22-23 (c'est l'édition que Girodet possédait dans sa bibliothèque).

9. Mentionné d'après D. et G. Wildenstein, Documents complémentaires au catalogue de l'œuvre de Louis David, Paris, La Bibliothèque des Arts, 1975, p. 158.

10. F.-R. de Chateaubriand, Le Génie du christianisme ou Beautés de la religion chrétienne, édition établie par M. Regard, Paris, Gallimard, « Bibliothèque de la Pléiade », 1978, p. 873. 
11. L'exil est, de même que la mort, l'un des thèmes fondamentaux du récit, cf. D. T. Kadish, "Symbolism of exile: The Opening Description in Atala ", dans The French Review, février 1982, p. 358-366.

12. F.-R. de Chateaubriand, Atala ou les amours des sauvages dans le désert, dans Atala René Les Natchez, introduction, commentaire et notes de J.-C. Berchet, Paris, Librairie générale française, 1989, p. 102, dorénavant ARL.

13. Ibid., p. 151.

14. C'est moi qui souligne.

15. Ibid., p. 123.

16. Ibid., p. 133.

17. ARL, p. 145. C'est moi qui souligne.

18. Ibid., p. 150.

19. C'est moi qui souligne.

20. F.-R. de Chateaubriand, Le Génie du Christianisme, cit., p. 796. Cf. S. Guéguan, op. cit., p. 150.

21. Ibidem.

22. Ibidem.

23. Comme le soulignait déjà A. Poirer, Les idées artistiques de Chateaubriand, Paris-Limoges, PUF, 1930 , p. 34.

24. Cf. E. Pottier, «Thanatos et quelques représentations funéraires sur des lécythes blancs attiques", dans Monuments et Mémoires publiés par l'Académie des Inscriptions et Belles-Lettres, premier fascicule, t. XXII, Paris, 1916.

25. G. E. Lessing, Du Laocoon ou des limites de la poésie et de la peinture, Paris, Renouard, 1802, p. 359.

26. A. Poirer, op. cit., p. 44 et sq.

27. Bernardin de Saint-Pierre, Études sur la nature, Paris, Didot, 1784, III, p. 288.

28. C. Savettieri, " "Il avait retrouvé le secret de Pygmalion" : Girodet, Canova e l'illusione della vita ", dans Studiolo, n. 2, 2003, p. 20-23. Chateaubriand, qui connut Canova en 1803, appréciait lui aussi sa sculpture, cf. F. Mazzocca, « Chateaubriand e il mito di Canova », dans M. Ariani (dir.), La parola e l'immagine. Scritti in onore di Gianni Venturi, Firenze, Olschki, 2011, p. 219-229.

29. Girodet possédait l'édition de 1766 de l'Histoire de l'art des anciens (cf. V. Bajou et S. LemeuxFraitot, Inventaire après décès de Gros et Girodet, Paris, Imprimerie Inuméric, 2002, n. 40, p. 388) qui ne fut pas reconnue par l'auteur ; cependant puisqu'il s'agit de la source utilisée par le peintre je donne ici la référence aux pages de cette édition: J.J. Winckelmann, Histoire de l'art chez les anciens ouvrage traduit de l'allemand par Gottfries Sellius et rédigé par Jean-Baptiste René de Robinet, Paris, 1766, I, p. 254-256 ; II, p. 29-31, 284-285.

30. [Auteur non signé], Première journée d'an Cadet Buteux au Salon de 1808, Paris, Aubry, 3 décembre 1808, notice « M. Girodet », n. 258.

31. [Auteur non signé], Observations sur le salon de l'an 1808 Tableaux d'histoire, Gueffieret et Delaunay, Paris, 1808, dans « Collection Deloynes » (Bibliothèque Nationale de France), vol. 43, n. 1139.

32. [Auteur non signé], « Salon d'exposition », dans Le Courrier de l'Europe, 27 novembre 1808.

33. A. M. G., « Beaux-Arts. Salon de 1808 », dans Le Publiciste, 28 octobre 1808.

34. Cité. d'après D. et G. Wildenstein, op. cit., p. 158.

35. Cité d'après J. Pruvost-Auzas, Girodet 1767-1824, Catalogue Exposition, Montargis, Musée Girodet, 1967, n. 133.

36. C. Savettieri, « "Il avait retrouvé le secret de Pygmalion": Girodet, Canova e l'illusione della vita ", cit., p. 14-42.

37. Voir par exemple le poète Ugo Foscolo, Epistolario, édition par P. Carli, Firenze, Le Monnier, 1954, IV, p. 134-135; p. 177-178.

38. A. Shelton, «Girodet et Boutard. Portrait d'une alliance artistico-journalistique au temps de Napoléon », dans S. Bellenger (dir.), Girodet, cit., p. 129-136. 
39. M.B [J. B. Boutard], « Beaux-Arts. Salon de 1808 », Feuilleton du Journal de l'Empire, 19 novembre 1808, p. 1-4.

40. G. Levitine, Some unexplored aspects of the Illustrations of Atala, cit., p. 139-144.

41. J. F. Moffitt, op. cit., p. 123-126.

42. Examen critique et raisonné des tableaux des peintres vivans formant l'exposition de 1808, Paris, Hocquart, 1808, n. 258, p. 10.

43. M. Gourdin-Servenière, « Le désir de la mort dans René et Les Natchez. Constantes du tourment romantique de l'âme", dans G. Erns (dir.), La mort en toutes les lettres, Nancy, Presses Universitaires de Nancy, 1983, p. 109-120.

44. F.-R. de Chateaubriand, Atala, cit., p. 158.

45. C. Savettieri, Ingannare la morte. Anne-Louis Girodet e l'illusione dell'arte, cit., p. 31-36.

46. Ibid., p. 16-17.

47. Ibid., p. 55 et sq.

48. Les Veillées est un poème de Girodet dont la rédaction commença avant 1801, et continua au moins jusqu'en 1807. Comme la plupart des écrits de Girodet, ce poème est resté manuscrit et publié post mortem par P. A. Coupin, Euvres posthumes de Girodet, Paris, Renouard, 1829. On ne connaît pas la destinée des manuscrits originaux, cependant les copies manuscrites de ces documents rédigées par Coupin sont consultables aux Archives Départementales des Yvelines (Versailles). Ces copies, qui transcrivent les textes de Girodet, présentent des coupures et des corrections apportées par Coupin et intégrées dans l'édition des Euvres posthumes. Ces copies permettent donc de reconstruire les textes originaires. Cf. C. Savettieri, Ingannare la morte. AnneLouis Girodet e l'illusione dell'arte, cit., p. 14-15.

49. A. L. Girodet, Deuxième Veillée, Archives Départementales des Yvelines, J2073, p. 18r-19v. : « Au terrible ils ont su marier la beauté. / Ils n'ont point fait marcher un dégoûtant squelette: / Une beauté parfaite / Doucement endormie au faite d'un tombeau, / Un génie attristé renversant son flambeau, / Un papillon mourant, une rose flétrie, / Exprimaient moins la mort qu'un départ de la vie. Emblème simple et vrai, même consolateur / qui compare nos jours aux instans d'une fleur $»$.

50. A.-L. Girodet, Sixième veillée, ibid., p. 8.

51. La transcription manuscrite de Coupin présente en ce lieu plusieurs biffures, et la version plus lisible est Naim, un nom qui ne correspond à aucun personnage ni à aucun lieu d'un texte littéraire où l'on pourrait retrouver une telle description : très probablement Coupin n'arrivait pas à déchiffrer le mot écrit par Girodet et l'a interprété ainsi.

52. A.-L Girodet, Dissertation sur l'amour et les grâces, Archives Départementales des Yvelines, J 2075 , c. 57.

53. Ch. Baudelaire, «Exposition universelle de 1855 », dans Ch. Baudelaire, Écrits sur l'art, Paris, Gallimard, 1971, I, p. 386-387.

\section{INDEX}

Mots-clés : Girodet (Anne Louis), Chateaubriand (François-René de), grâce, beau idéal, mort, Lessing (Gotthold Epraim), Winckelmann (Johann Joachim) 